\title{
Diethylnitrosamine-Induced Spongiosis Hepatis in Medaka, Oryzias Latipes
}

\author{
W.N. Norton* and Henry S. Gardner** \\ * Biology Department, Southeastern Louisiana University, Box 10736, Hammond, LA 70402 \\ ** US Army Biomedical Research \& Development Laboratory, Fort Detrick, Frederick, MD \\ 21701-5010
}

The Japanese medaka, Oryzias latipes has served as a popular fish model for carcinogenicity studies, several of which have included diethylnitrosamine (DENA), a site-specific carcinogen for hepatic neoplasms [1]. The primary objective of this investigation was to obtain ultrastructural information regarding DENA-induced spongiosis hepatic, a hepatic lesion characterized by mutilocular cyst-like complexes in the Japanese medaka, a small aquarium fish. A group of 8 dayold embryos was treated continuously with $3000 \mathrm{mg} / 1$ of DENA for 48 hours. At 50 weeks subsequent to the end of the exposure, livers were excised and processed for scanning and transmission electron microscopy. Spongiosis hepatis was detected in 8 of the 14 fish exposed to DENA, while smaller but comparable lesions were observed in 2 of the 6 control fish.

A discrete manifestation of exposure to DENA for the majority of medaka investigated was the formation of spongiosis hepatis, a multiocular cyst-like lesion (Fig.1). Cells that formed the framework of spongiotic lesions were similar in appearance to perisinusodial cells. They were characterized by small irregular nuclei and elongated cytoplasmic processes that formed desmosomes at cellular junctions (Fig. 2). Unlike the perisinusoidal cells associated with sinusoids of control liver, the attenuated cells that formed spongiotic lesions were characterized by pronounced numbers of coated and uncoated vesicles, increases in the number of ribosomes and a relatively high ratio of euchromatin to heterochromatin. There was no evidence of collagen or basement membrane-like material in the cystic zones. Necrotic cells and fibrous or flocculent material were observed in a majority of cystic cavities (Fig. 3). The material was periodic acidSchiff positive. All spongiotic lesions displayed uncharacterized cells that were distinguished by the presence of electron dense bodies, many of which contained filaments (Fig. 4). Recognizable hepatocytes associated with the spongiotic lesions demonstrated several features of cytotoxicity.

Results of this ultrastructural investigation indicate that DENA and/or its metabolites induce chronic aberrations in cells associated with the spongiotic lesions. Discernible increases in both the number of ribosomes and euchromatin content suggest exposure to the hepatocarcinogen promotes protein synthesis and gene activity. The prolonged organizational and cellular changes that persist in regions of spongiosis hepatic and the apparent lack of regeneration reflect the chronic cytotoxic nature of DENA. Several of the cytoplasmic features observed in this study are consistent with the cellular changes that are associated with the formation of preneoplastic foci found in livers exposed to hepatocarcinogens [2].

\section{References}

[1] D.E. Hinton et al., Aquatic Toxicol. 11 (1998) 77.

[2] J.A. Couch and L.A. Courtney, J. Natl. Cancer Inst. 79 (1987) 297. 


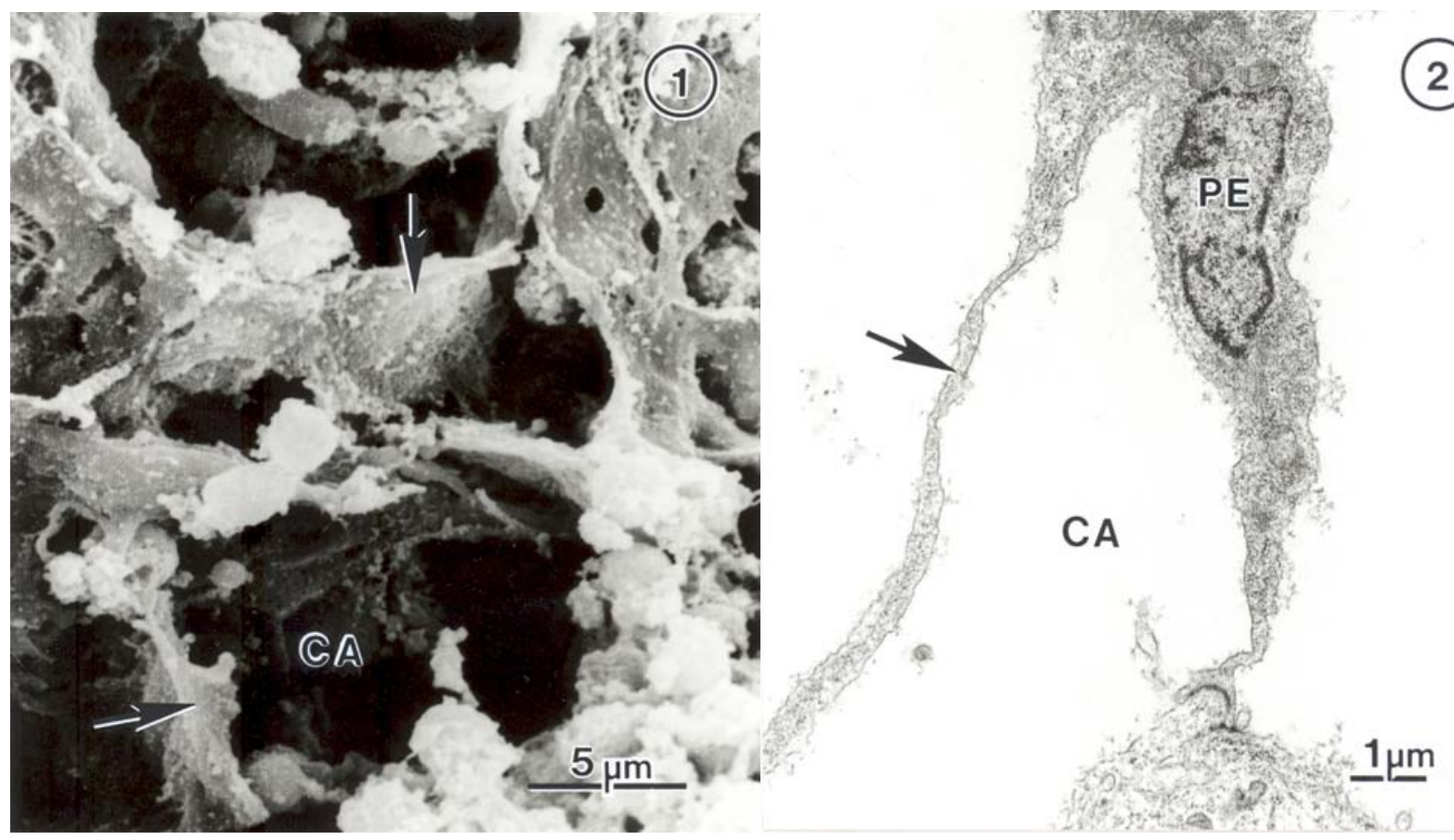

Fig. 1. Spongiosis hepatic is distinguished by pronounced multiocular cyst-like cavities (CA) which are bordered by attenuated cells (arrows).

Fig. 2. The structural foundation of spongiosis hepatis consists of perisinusoidal cells (PE) that possess elongated cytoplasmic extensions (arrow) which form the border of cavities (CA).

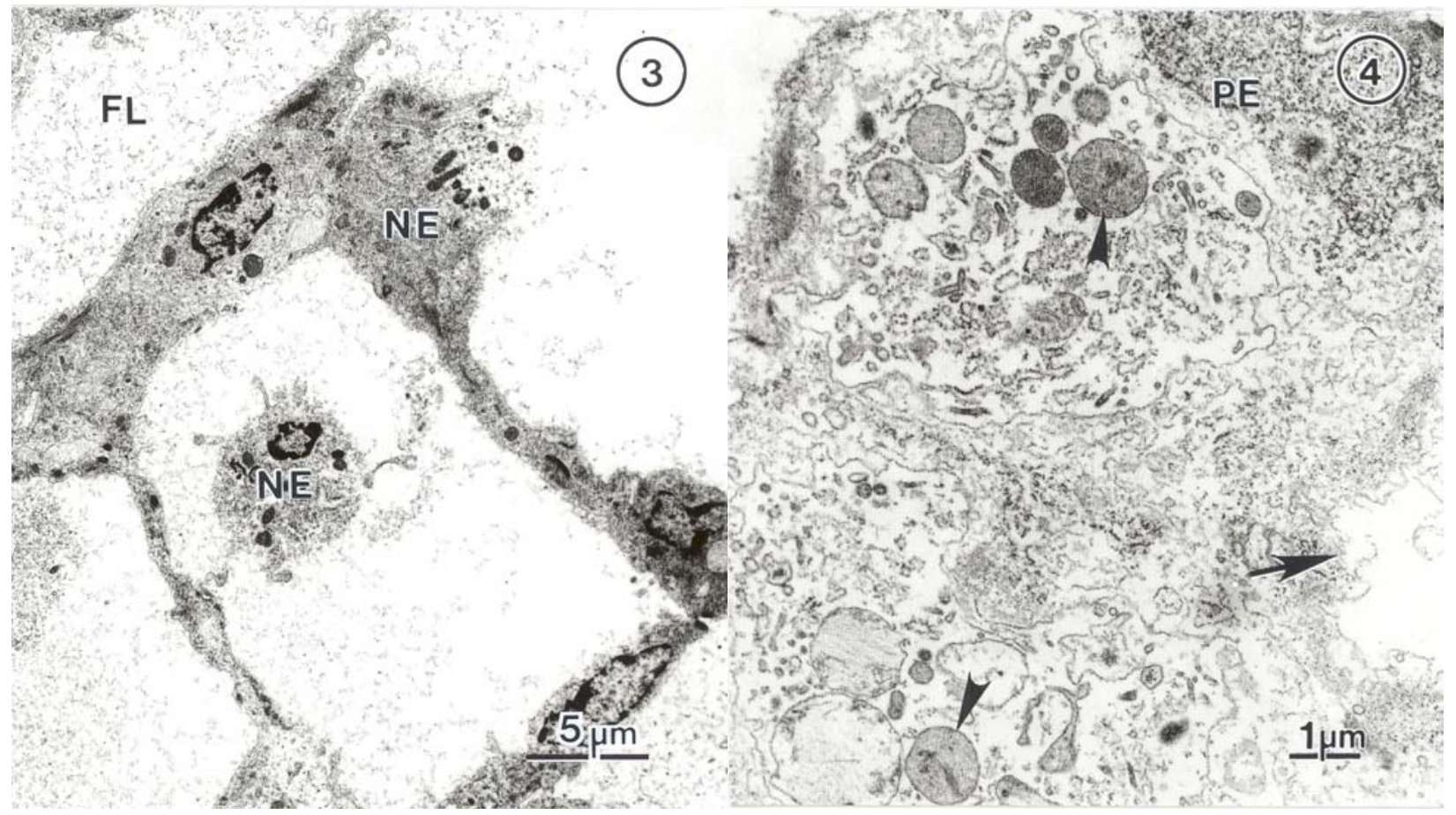

Fig. 3. Several necrotic cells (NE), none of which are structurally similar to hepatocytes, fragment with the dissipated cellular debris appearing as flocculent material (FL) in cavities.

Fig. 4. Electron-dense bodies (arrowheads) and fragmented membranes (arrow) are characteristic features of necrotic cells associated with spongiotic lesions. 